\title{
Suppressing Fermi acceleration in two-dimensional driven billiards
}

\author{
Edson D. Leonel ${ }^{1,2}$ and Leonid A. Bunimovich ${ }^{2}$ \\ ${ }^{1}$ Departamento de Estatística, Matemática Aplicada e Computação-IGCE-UNESP-Univ Estadual Paulista, \\ Avenida 24A, 1515 Bela Vista, CEP 13506-700_Rio Claro, SP, Brazil \\ ${ }^{2}$ School of Mathematics, Georgia Institute of Technology, Atlanta, Georgia 30332-0160, USA
}

(Received 3 December 2009; published 2 July 2010; corrected 7 July 2010)

\begin{abstract}
We consider a dissipative oval-like shaped billiard with a periodically moving boundary. The dissipation considered is proportional to a power of the velocity $V$ of the particle. The three specific types of power laws used are: (i) $F \propto-V$; (ii) $F \propto-V^{2}$ and (iii) $F \propto-V^{\delta}$ with $1<\delta<2$. In the course of the dynamics of the particle, if a large initial velocity is considered, case (i) shows that the decay of the particle's velocity is a linear function of the number of collisions with the boundary. For case (ii), an exponential decay is observed, and for $1<\delta<2$, an powerlike decay is observed. Scaling laws were used to characterize a phase transition from limited to unlimited energy gain for cases (ii) and (iii). The critical exponents obtained for the phase transition in the case (ii) are the same as those obtained for the dissipative bouncer model. Therefore near this phase transition, these two rather different models belong to the same class of universality. For all types of dissipation, the results obtained allow us to conclude that suppression of the unlimited energy growth is indeed observed.
\end{abstract}

DOI: 10.1103/PhysRevE.82.016202

PACS number(s): 05.45.Pq, 05.45.Tp

\section{INTRODUCTION}

The systems where particles do not interact with each other and move freely experiencing elastic collisions from the boundary are called billiards [1]. Billiard type systems demonstrate regular [1], mixed [2] or fully chaotic behavior [3], and are used in many physical experiments including reflection of light from mirrors [4], superconducting [5] and confinement of electrons in semiconductors by electric potentials $[6,7]$, waveguides [8,9], microwave billiards $[10,11]$, ultracold atoms trapped in a laser potential [12-15] and also mesoscopic quantum dots [16].

The famous phenomenon of Fermi acceleration (FA) [17] was studied in various billiardlike models [18-23] where the boundary moves in time, i.e., it is time dependent. The introduction of time dependence into the boundary yields the particle to change energy upon collision. If the collision is headon/tail-on then the particle gains/loses energy. A key question addressed in studies on FA [17] is whether a particle's energy can grow to infinity. The answer to this question is not trivial and depends on the geometry of the boundary and the kind of time perturbation. The Loskutov-Ryabov-Akinshin (LRA) conjecture [24] claims that if the dynamics of the particle is chaotic while the boundary is static, thus this is a sufficient condition to observe FA when a time perturbation to the boundary is introduced. It has been shown recently [25] that a time-dependent elliptic billiard can also generate FA, therefore, the answer could also be "yes" even for (some) integrable billiards.

In this paper, we are concerned with the mechanisms of suppressing FA. We therefore study the dynamics of an ensemble of non interacting particles in a time-dependent ovallike shaped billiard. It is assumed that the particles experience a drag force which is proportional to a power of the particle's velocity. The reflection law is not modified and the particles still keep moving along straight lines, as in standard billiards. However, the velocity of each particle is not a con- stant anymore and decreases as the particle moves. Depending on the kind of damping force considered, the particles might eventually have all their energy dissipated, leading them to reach the state of rest, thus, stopping the dynamics. Our main goal in this paper is to investigate the process of competition between the FA and dissipation of the particle energy via a drag force. We specifically address the question whether FA is observed under the presence of the drag force proportional to a power of the particle's velocity. Our numerical results show convincingly that FA is suppressed, even in the regime of small dissipation. Particularly and depending on the type of damping force, the dissipation leads the particles to reach the state of rest. Recently, inelastic collisions have been considered in a time-dependent version of an oval billiard and the results confirm that FA was also suppressed [26]. We have made a similar investigation in a driven elliptic billiard [27] which led also in suppressing FA. Additionally, a new writing of the LRA conjecture was proposed in Ref. [27]. These results allow us to conjecture that FA is not a structurally stable phenomenon.

This paper is organized as follows. In Sec. II, we present the model and derive the equations for the mapping that fully describes the dynamics of the particle. Section III deals with the numerical results for all types of damping forces. Final remarks and conclusions are presented in Sec. IV.

\section{MODEL AND THE MAPPING}

The model considered consists of a classical particle confined to a domain with the boundary moving in time according to the following equation in polar coordinates

$$
R(\theta, \epsilon, a, t, p)=1+\epsilon[1+a \cos (t)] \cos (p \theta),
$$

where $\epsilon$ is the amplitude of the circle's perturbation, $a$ is the amplitude of the time perturbation, $\theta$ is the angular coordinate, $t$ is the time and $p>0$ is an integer. The variation of the control parameters let us obtain different kinds of geometry. 


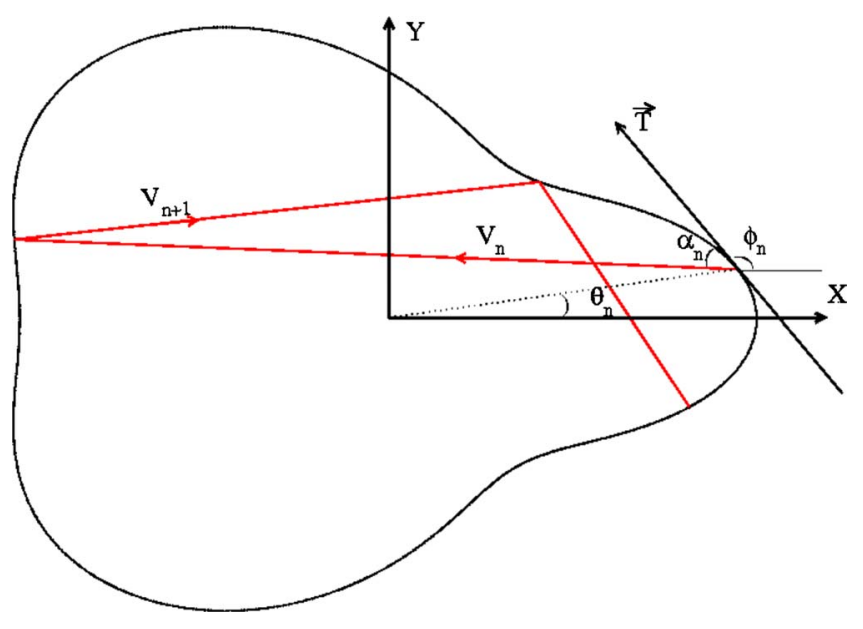

FIG. 1. (Color online) Illustration of the coordinate angles in the oval-like shaped billiard for three snapshots of a typical orbit.

If $\epsilon=0$ we get the circular billiard. The phase space is then foliate into invariant curves and no chaos is present [1]. If $\epsilon \neq 0$ and $a=0$, then for $\epsilon<\epsilon_{c}=1 /\left(p^{2}+1\right)$, the phase space contains both periodic islands, invariant spanning curves corresponding to rotating orbits (which will denote here only as invariant tori) and chaotic regions [2,28] while for $\epsilon \geq \epsilon_{c}$ all the invariant tori are destroyed [29] but then KolmogorovArnold-Moser (KAM) islands may survive. For $a \neq 0$ the particle can gain or lose energy upon collisions with the boundary. According to the LRA conjecture [24] (see also Ref. [27] for an extended LRA conjecture), if the phase space has a chaotic region while the boundary is static, the introduction of time perturbation to the boundary is a sufficient condition to produce FA. Recent results demonstrated that the oval billiard [30,31] does indeed produces FA. Such a phenomenon was also observed for specific perturbation of the elliptical billiard [25]. Since it is known [30,31] that FA is observed in driven oval-like shaped billiards and the LRA conjecture holds for this system, our goal in this paper is to analyze whether a dissipative force of a drag-type can suppress such a phenomenon.

The dynamics of the model is described by an implicit four-dimensional (4D) mapping. Given an initial condition $\left(\theta_{n}, \alpha_{n}, V_{n}, t_{n}\right)$, where $\alpha_{n}$ is the angle between the trajectory of the particle and the tangent line at the point of the boundary with angular coordinate $\theta_{n}, V_{n}>0$ is the velocity of the particle and $t_{n}$ is the time at the $n^{\text {th }}$ collision of the particle with the boundary, the corresponding mapping is $T\left(\theta_{n}, \alpha_{n}, V_{n}, t_{n}\right)=\left(\theta_{n+1}, \alpha_{n+1}, V_{n+1}, t_{n+1}\right)$. Figure 1 shows three snapshots of a typical orbit and the coordinate angles for the oval-like shaped billiard.

We consider three different laws for the damping force acting on the particle, namely: (i) $F=-\eta^{\prime} V$; (ii) $F=-\eta^{\prime} V^{2}$ and (iii) $F=-\eta^{\prime} V^{\delta}$ with $\delta \neq 1$ and $\delta \neq 2$ where $\eta^{\prime}$ is the viscosity coefficient which is assumed to be constant along the particle's trajectory. Since this model is two dimensional, these particular choices were made to allow a direct comparison with results obtained for one-dimensional model [32,33]. It is interesting to stress that other different kinds of forces proportional to the particle's velocity have also been considered in the literature. In particular, if the particle is moving under the action of a magnetic field [34,35] (see also Ref. [36] for recent results), it will move along of Larmor arcs of radius $R$ which are proportional to the particle's velocity. One of the most important properties of such kind of perturbation is that time-reversal symmetry is broken [34]. Such kind of force however is qualitatively different from the type we are considering in this paper, which addresses dissipation properly.

The exact expressions for the mapping will be obtained for the case (i). To obtain the equation that describes the velocity of the particle along its trajectory, the second Newton's law of motion must be solved. After a direct integration of $-\eta^{\prime} V=m d V / d t$ with the initial velocity $V_{n}>0$, we obtain the equation $V(t)=V_{n} \exp \left[-\eta\left(t-t_{n}\right)\right]$, with $\eta=\eta^{\prime} / m$ and $t$ $\geq t_{n}$. A displacement of the particle along a straight line is obtained by an integration of $d r / d t=V(t)$, yielding $r(t)$ $=V_{n}\left\{1-\exp \left[-\eta\left(t-t_{n}\right)\right]\right\} / \eta$ for $t \geq t_{n}$. Therefore, the coordinates of the particle are $X(t)=R\left(\theta_{n}, t_{n}\right) \cos \left(\theta_{n}\right)+r(t) \cos \left(\phi_{n}\right.$ $\left.+\alpha_{n}\right)$ and $Y(t)=R\left(\theta_{n}, t_{n}\right) \sin \left(\theta_{n}\right)+r(t) \sin \left(\phi_{n}+\alpha_{n}\right)$ with $\phi_{n}$ $=\arctan \left[Y^{\prime}\left(\theta_{n}, t_{n}\right) / X^{\prime}\left(\theta_{n}, t_{n}\right)\right]$ and $X^{\prime}=d X / d \theta$ and $Y^{\prime}$ $=d Y / d \theta$. Two different situations may occur after the particle suffers a collision with the boundary and leaves the collision zone [region on the plane circumscribed by $R(\theta) \leq R_{c}(\theta)$ $=1-\epsilon(1+a)]$ : (a) the particle has enough energy to enter the collision zone again $\left(R \geq R_{c}\right)$ and has another collision with the boundary or; (b) the particle does not have enough energy to reach a point of the next collision and, thanks to the dissipation, the particle stops, thus, having a maximum displacement $r_{\max }=V_{n} / \eta$. The new angular coordinate $\theta_{n+1}$ is obtained, after evolving the dynamics of the particle by using molecular dynamics, as a root of the following equation

$$
\sqrt{X^{2}\left(t-t_{n}\right)+Y^{2}\left(t-t_{n}\right)}=1+\epsilon\left[1+a \cos \left(t-t_{n}\right)\right] \cos (p \theta),
$$

with $t \geq t_{n}$. The time at the next collision is obtained as

$$
t_{n+1}=t_{n}+\Delta t_{n}
$$

where $\Delta t_{n}=-\ln \left[1-\eta r\left(t_{c}\right) / V_{n}\right] / \eta$, with $t_{c}$ corresponding to the instant of the collision.

The reflection rules we consider for the collision of the particle with the boundary are the following:

$$
\begin{gathered}
\vec{V}_{n+1}^{\prime} \cdot \vec{T}_{n+1}=\vec{V}_{p}^{\prime}\left(t_{n+1}\right) \cdot \vec{T}_{n+1}, \\
\vec{V}_{n+1}^{\prime} \cdot \vec{N}_{n+1}=-\vec{V}_{p}^{\prime}\left(t_{n+1}\right) \cdot \vec{N}_{n+1},
\end{gathered}
$$

where the upper prime denotes that the velocities are measured with respect to the moving boundary reference frame. The vectors $\vec{T}$ and $\vec{N}$ are the unit tangent and normal vectors respectively and $\vec{V}_{p}\left(t_{n+1}\right)$ is the velocity of the particle immediately before the collision given by $\vec{V}_{p}\left(t_{n+1}\right)$ $=V_{n} \exp \left[-\eta\left(t_{n+1}-t_{n}\right)\right]$.

Based on Eqs. (4) and (5), the components of the velocity of the particle after a collision are

$$
\begin{aligned}
\vec{V}_{n+1} \cdot \vec{T}_{n+1}= & \left|\vec{V}_{p}\left(t_{n+1}\right)\right|\left[\cos \left(\alpha_{n}+\phi_{n}\right) \cos \left(\phi_{n+1}\right)\right] \\
& +\left|\vec{V}_{p}\left(t_{n+1}\right)\right|\left[\sin \left(\alpha_{n}+\phi_{n}\right) \sin \left(\phi_{n+1}\right)\right],
\end{aligned}
$$



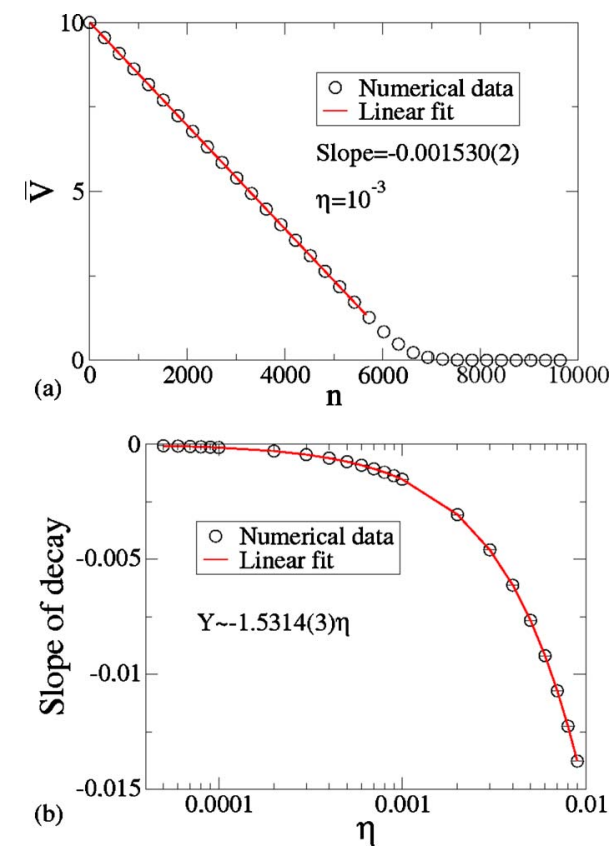

FIG. 2. (Color online) (a) Behavior of the average velocity as function of $n$. The control parameters used were $\epsilon=0.1, a=0.1, p$ $=3$, and $\eta=10^{-3}$ and the initial velocity was $V_{0}=10$. (b) Linear fit of the slope of the velocity's decay as function of $\eta$.

$$
\begin{aligned}
\vec{V}_{n+1} \cdot \vec{N}_{n+1}= & -\left|\vec{V}_{p}\left(t_{n+1}\right)\right|\left[\sin \left(\alpha_{n}+\phi_{n}\right) \cos \left(\phi_{n+1}\right)\right] \\
& -\left|\vec{V}_{p}\left(t_{n+1}\right)\right|\left[-\cos \left(\alpha_{n}+\phi_{n}\right) \sin \left(\phi_{n+1}\right)\right] \\
& +2 \frac{d R(t)}{d t}\left[\sin \left(\theta_{n+1}\right) \cos \left(\phi_{n+1}\right)\right] \\
& -2 \frac{d R(t)}{d t}\left[\cos \left(\theta_{n+1}\right) \sin \left(\phi_{n+1}\right)\right],
\end{aligned}
$$

where $d R / d t$ is the velocity of the moving boundary at the moment of collision. Then the velocity of the particle after the $(n+1)^{\text {th }}$ collision is

$$
V_{n+1}=\sqrt{\left(\vec{V}_{n+1} \cdot \vec{T}_{n+1}\right)^{2}+\left(\vec{V}_{n+1} \cdot \vec{N}_{n+1}\right)^{2}} .
$$

The angle $\alpha_{n+1}$ is obtained as

$$
\alpha_{n+1}=\arctan \left[\frac{\vec{V}_{n+1} \cdot \vec{N}_{n+1}}{\vec{V}_{n+1} \cdot \vec{T}_{n+1}}\right] .
$$

\section{RESULTS AND DISCUSSION}

We present and discuss in this section our results for all types of dissipation.

\section{A. Results for the case $F=-\eta V$}

The average velocity of the particle as function of $n$ is shown in Fig. 2(a). We started the simulation using the initial velocity $V_{0}=10$ while the initial angles $\alpha_{0} \in[0, \pi]$ and $\theta_{0}$ $\in[0,2 \pi]$ were chosen at random in a grid of $100 \times 100$ as well as the initial time $t_{0} \in[0,2 \pi]$. The control parameters used were $\epsilon=0.1, a=0.1, p=3$ and $\eta=10^{-3}$. With this particular set of control parameters, the boundary oscillates between $1-\epsilon[1+a]<R<1+\epsilon[1+a]$ and therefore, eventually, it changes the sign of the curvature. In the static case, such a change leads to the destruction of the invariant tori. So the simulations are done near a critical control parameter where the invariant tori are destroyed in the static case but some KAM islands still survive [29]. The average velocity of the particle was calculated using two different kinds of averages. The first one is the average over the time

$$
V_{j}=\frac{1}{n} \sum_{i=0}^{n-1} V_{i},
$$

while the other one is an ensemble average of Eq. (10)

$$
\bar{V}=\frac{1}{M} \sum_{j=1}^{M} V_{j},
$$

where $M$ denotes the number of different initial conditions (particles) in the ensemble. In this paper, all simulations were performed using $M=10^{4}$. As we see in Fig. 2(a), the average velocity decays linearly as a function of the number of collisions with the boundary. The slope of the decay was obtained via a linear fit as $-0.001530(2)$ where the term 2 $\times 10^{-6}$ represents the error of the fitting. Eventually, the particle reaches a critical velocity after leaving the collision zone and has no energy to reach the boundary again for the next collision and, after having all of its energy dissipated by the drag force, it stops. The plateau seen in Fig. 2(a) for $n$ $>7000$ represents few orbits which stay with low velocity after the linear decay is finished before stopping completely.

We have simulated the behavior of the decay of the particle's velocity for many different control parameters $\eta$. The slope of the decay as a function of the control parameter $\eta$ is shown in Fig. 2(b). It was shown analytically [32], that the behavior of the decay of the particle's velocity for the FermiUlam model (a classical particle bouncing between two rigid walls, where one of them is fixed and the other one moves periodically in time) is linearly dependent on the control parameter $\eta$, i.e., $V_{n} \propto V_{0}-2 n \eta$. It is worth mentioning that the Fermi-Ulam model is a one-dimensional (1D) system. In our case however, the dynamics might be considered as a generalization of the Fermi-Ulam model to two-dimensional (2D) and therefore, as shown in Fig. 2(b), the behavior of the average velocity of the particle is $V_{n} \propto V_{0}-1.5314(3) n \eta$. The results obtained allow to conclude that if the particle experiences a drag force proportional to its velocity, the phenomenon of unlimited energy growth (FA) is suppressed.

\section{B. Results for the case $F=-\eta V^{2}$}

The equation that needs to be integrated is $-\eta V^{2}=d V / d t$. Considering the initial velocity as $V_{n}>0$, we obtain that

$$
V(t)=\frac{V_{n}}{1+\eta\left(t-t_{n}\right)},
$$

with $t \geq t_{n}$. The integration of Eq. (12) gives the displacement of the particle 

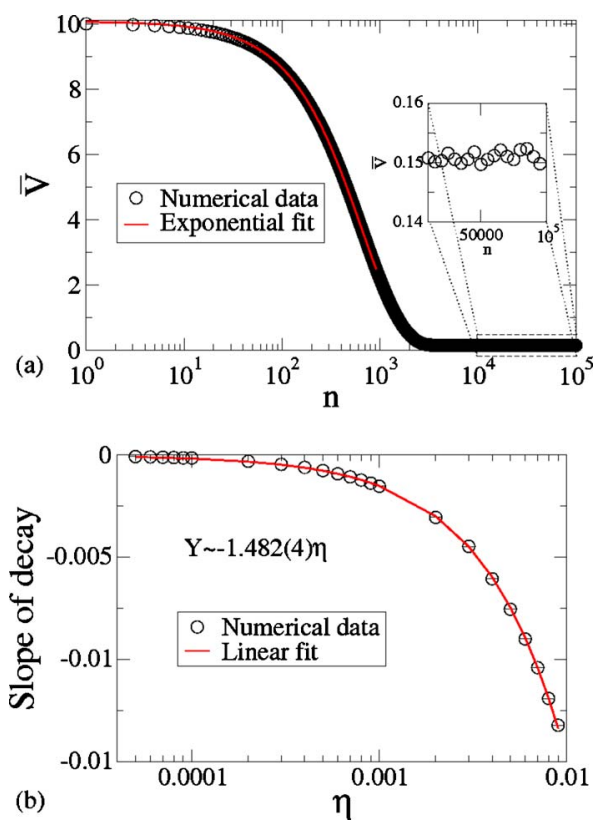

FIG. 3. (Color online) (a) The average velocity as function of $n$ for an initial $V_{0}=10$. The control parameters used were $\epsilon=0.1, a$ $=0.1, p=3$, and $\eta=10^{-3}$. (b) Linear fit of the slope of the velocity's decay as a function of $\eta$.

$$
r(t)=\frac{1}{\eta} \ln \left[1+\eta V_{n}\left(t-t_{n}\right)\right],
$$

for $t \geq t_{n}$. Updating the mapping with Eqs. (12) and (13), we can then follow the dynamics of the particle. The behavior of $\bar{V}$ as function of $n$ is shown in Fig. 3(a). For a large initial velocity, say $V_{0}=10$, the velocity of the particle experiences an exponential decay for a short time and then, after reaching a characteristic crossover, say $n_{c}$, the velocity bends toward a regime of a constant plateau. Contrary to the case of $F \propto-V$, the dissipation does not stop the dynamics of the particle since $r(t)$ is a function which grows in time. Therefore, the particle keeps moving and the dissipation gets smaller as the velocity of the particle diminish. Furthermore, if the particle has escaped the collision zone, it enters it again and suffers another collision with the boundary. An exponential fitting for the decay shown in Fig. 3(a) gives that $\bar{V}$ $=V_{0} \exp [-0.00153(1) n]$ for the control parameter $\eta=10^{-3}$. We performed the simulations for many different control parameters and obtained the slope of the decay for each one of them. Figure 3(b) shows the behavior of the slope of the decay as a function of the control parameter $\eta$. A linear fitting gives the slope $\propto-1.482(4) \eta$. A comparison with the Fermi-Ulam model can also be made. As discussed in [33], the decay of the velocity in the Fermi-Ulam model was obtained analytically as $V_{n}=V_{0} \exp [-2 n \eta]$. However, in the time-dependent oval billiard, we have found that $V_{n}$ $=V_{0} \exp [-1.482(4) n \eta]$. Therefore, the decay obtained for the oval billiard is slower for both cases (i) and (ii) what is probably associated with the dimensionality of the system. This difference might also be caused by the fact that there is now a competition between production and suppression of FA, while Fermi-Ulam model does not exhibit FA at all.
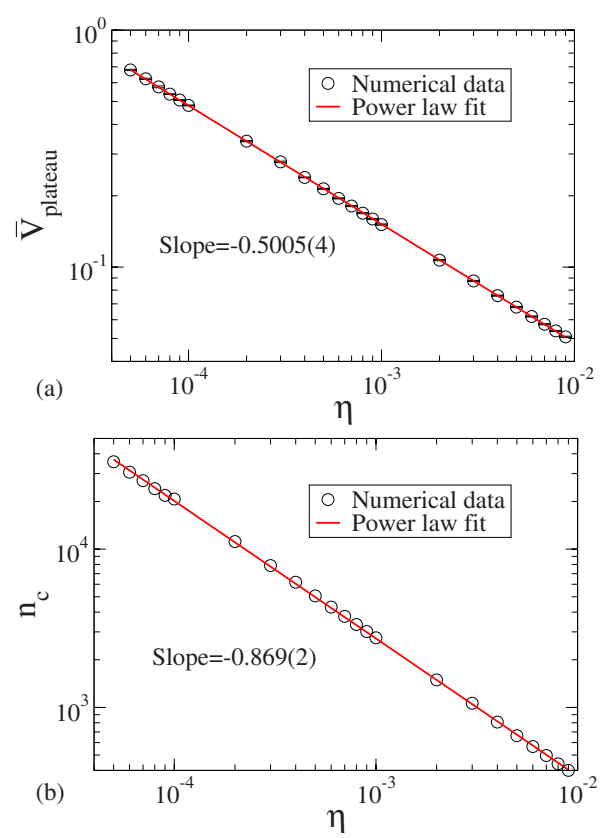

FIG. 4. (Color online) (a) The average velocity along the plateau for large $n$ as a function of the control parameter $\eta$. The control parameters used were $\epsilon=0.1, a=0.1$, and $p=3$. (b) Plot of $n_{c}$ as a function of $\eta$.

Consider now the behavior of the average velocity for large values of $n$, i.e., along the constant plateau. The zoom in Fig. 3(a) shows few points along the constant plateau. We see that it fluctuates around an average value and does not decreases to zero. But the question is: how changes the average velocity along the plateau if the strength of the dissipation is changed? If a reduction takes place, it is expected that dissipation affects the FA less and, therefore, the constant plateau should rise. It does indeed happens. The behavior of $V_{\text {plateau }}$ as function of $\eta$ is shown in Fig. 4(a). A power law fitting gives us that $V_{\text {plateau }} \propto \eta^{-0.5}$. Thus, as the control parameter $\eta \rightarrow 0$, the velocity diverges, recovering FA. This behavior characterizes a smooth transition from suppression to production of FA. Figure 4(b) shows the behavior of the crossover iteration number $n_{c}$, which is the number of collisions with the boundary needed to change the regime of decay to the regime of constant velocity, for the initial velocity $V_{0}=10$. The ensemble used was a grid of $100 \times 100$ for the variables $\alpha_{0} \in[0, \pi] \times \theta_{0} \in[0,2 \pi]$. A power law fitting applied to Fig. 4(b) yields $n_{c} \propto \eta^{-0.869(2)}$.

We now discuss the behavior of the average velocity of the particle when a given initial velocity is very small compared to the maximum component of the moving boundary velocity. The behavior of the average velocity as function of $n$ is shown in Fig. 5(a). One can clearly see that the average velocity starts growing for small $n$ and then, after reaching a critical crossover $n_{x}$, it bends toward a regime of saturation, marked by a constant plateau. As the damping coefficient decreases, the average velocity can reach higher values. Moreover the crossover also rises. We then suppose that by 

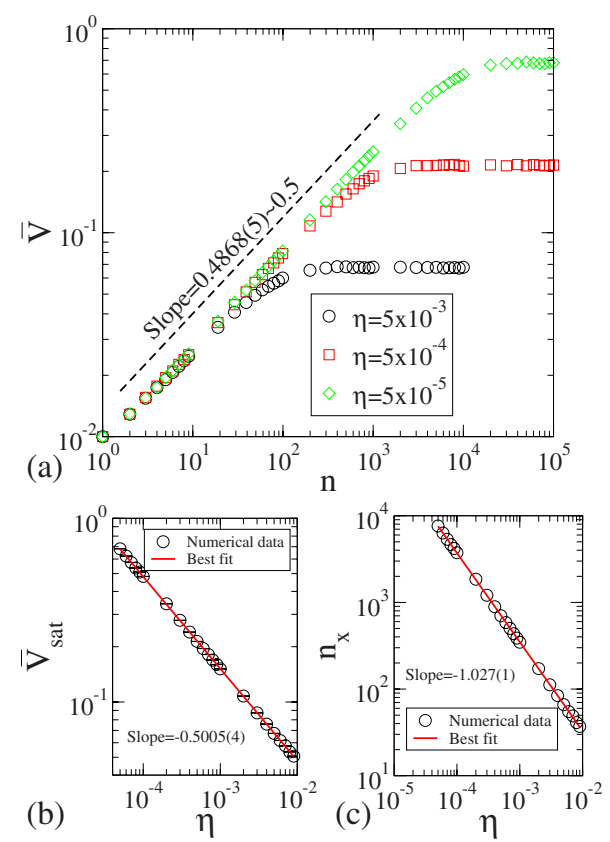

FIG. 5. (Color online) (a) The average velocity as function of $n$ for three different control parameters $\eta$, as labeled in the figure. The initial velocity used was $V_{0}=10^{-2}$ and the control parameters were $\epsilon=0.1, a=0.1$ and $p=3$. (b) Plot of $\bar{V}_{\text {sat }}$ versus $\eta$. A power law fitting yields the slope $\alpha=-0.5005(4)$. (c) Plot of $n_{x}$ versus $\eta$. The slope obtained is $z=-1.027(1)$.

$$
\bar{V} \propto n^{\beta},
$$

where $\beta$ is a critical exponent;

(ii) For very large $n$, i.e., $n \gg n_{x}$, the average velocity is written as

$$
\bar{V}_{\text {sat }} \propto \eta^{\gamma},
$$

and $\gamma$ is also a critical exponent;

(iii) Finally, the crossover $n_{x}$, which marks the change from the regime of growth to the saturation is given by

$$
n_{x} \propto \eta^{z}
$$

where $z$ is a critical exponent.

Using the formalism shown in $[37,38]$, it is possible to describe the behavior of $\bar{V}$ using a scaling function. The critical exponents are obtained by numerical fittings as shown in Figs. 5(b) and 5(c), and the obtained values were $\beta$ $=0.4868(5) \cong 0.5, \quad \gamma=-0.5005(4) \cong-0.5$, and $z=-1.027(1)$ $\cong-1$. Using these three values, we can rescale the axis and obtain a single and universal plot, as shown in Fig. 6.

We now discuss the critical exponents and the transition. First, it is important to mention that, as the damping coefficient $\eta \longrightarrow 0$, the unlimited energy growth is recovered, as described by Eq. (15) given that $\gamma=-0.5$. Since FA is recovered, the crossover $n_{x}$ also diverges for $\eta \rightarrow 0$, as easily checked from Eq. (16) for $z=-1$. The critical exponents obtained for the phase transition from limited to unlimited

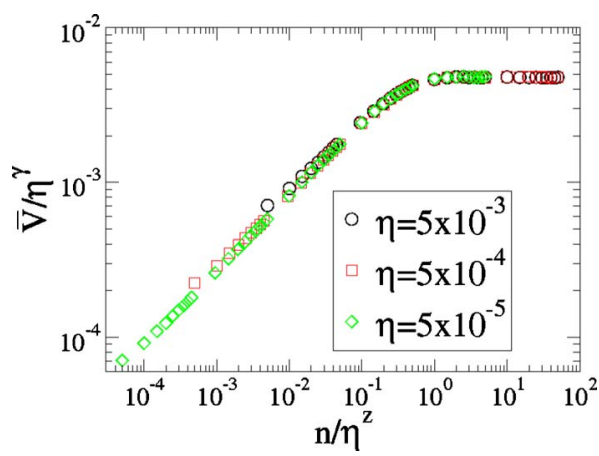

FIG. 6. (Color online) Rescaled axis showing a single and universal plot of three different curves of $\bar{V}$. The control parameters used are labeled in the figure.

energy growth in this section are the same as those obtained from the dissipative bouncer model $[37,38]$ (a classical particle suffering inelastic collisions with a periodically moving wall in the presence of a constant gravitational field). Despite the two models are very different, e.g., the bouncer model is a $1 \mathrm{D}$ system and the particle experiences the action of a constant gravitational field, while the driven oval-like shaped billiard is a 2D system, the dissipation used in the bouncer model is qualitatively different from the dissipation used in this section, the phase transition from limited to unlimited energy exhibits the same critical exponents. Therefore the two models belong to the same class of universality near this phase transition. In principle the classification of universality classes is made only using the critical exponents $\gamma, \beta$, and $z$.

If one think a little bit deeper in the dynamics, when the restitution coefficient in the bouncer model approaches the unity, the dissipative dynamics is replaced by nondissipative one and chaotic attractors no longer exist anymore. The interval of time between impacts is then proportional to the particle's velocity $(\Delta t \propto V)$. With increasing velocity the time between collisions increases too leading to a loss of correlation between the impacts. This leads to an increase of randomness in the system therefore producing unlimited energy growth. In the oval-like billiard, when the damping coefficient approaches zero (no dissipation), as opposite to the bouncer model, the time between impacts is inversely proportional to the particle's velocity $\left(\Delta t \propto V^{-1}\right)$. For fixed boundary there exist a smooth transition from dissipative to conservative dynamics [28], thus, leading the phase space in the angular coordinates to show mixed form [28,29]. Such a mixed structure however produces conservative chaotic portions in the phase space that is what the LRA conjecture claims [24] as needed to observe FA when time perturbation to the boundary is introduced. Basically chaotic dynamics works more like stochastic behavior yielding in diffusion in the average velocity and hence leading the particle to experience FA. Despite the differences between the two models and the different mechanisms which produce FA, the behavior of the average velocity near a transition from dissipative to no dissipative dynamics is described by the same law with the same critical exponents. 

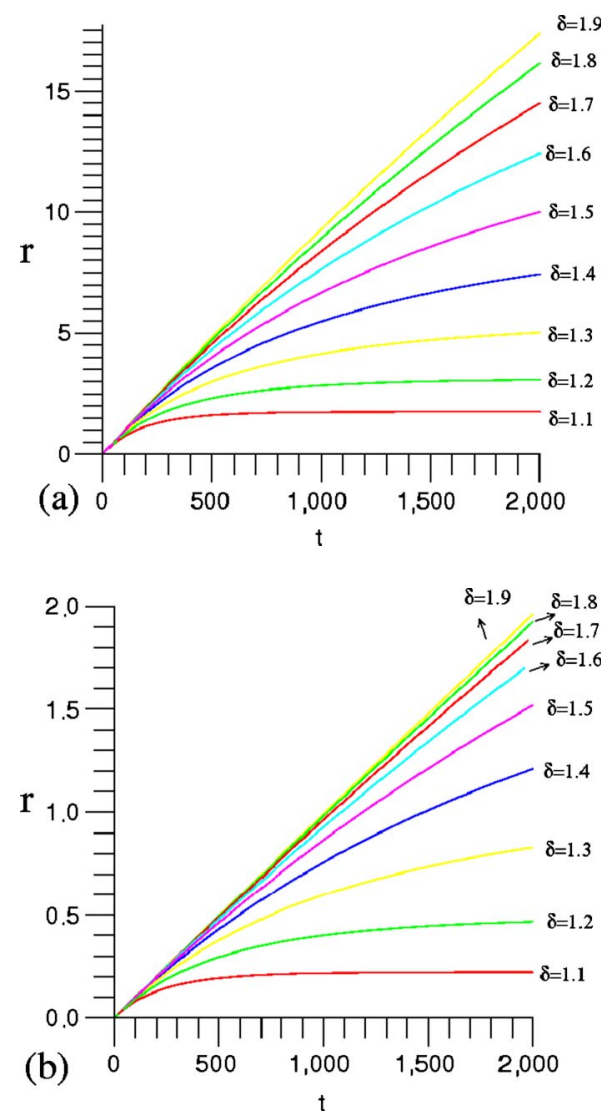

FIG. 7. (Color online) (a) Plot of $r$ versus $t$ for different values of the exponent $\delta$, as shown in the figure. The initial velocity used was $V_{0}=10^{-3}$. (b) Same plot of (a) for initial velocity $V_{0}=10^{-2}$.

\section{Results for the case $F=-\eta V^{\delta}$}

In this section, we consider the dissipation $F=-\eta V^{\delta}$ with $1<\delta<2$, acting in the particle. Taking the initial velocity as $V_{n}>0$ and integrating the equation of motion we obtain

$$
V(t)=\left[V_{n}^{1-\delta}-\eta(1-\delta)\left(t-t_{n}\right)\right]^{1 / 1-\delta},
$$

with $t \geq t_{n}$ and $\delta \neq 1$. The displacement of the particle is therefore obtained by the integration of $d r / d t=V(t)$, which gives

$$
r(t)=\frac{V_{n}^{2-\delta}}{\eta(2-\delta)}-\frac{\left[V_{n}^{1-\delta}-\eta(1-\delta)\left(t-t_{n}\right)\right]^{2-\delta / 1-\delta}}{\eta(2-\delta)},
$$

with $\delta \neq 1, \delta \neq 2$ and $t \geq t_{n}$. Depending on the control parameter $\delta$, the dissipation can lead to a complete stopping of the particle. We illustrate the typical regimes of the displacement of the particle in Figs. 7(a) and 7(b)by some plots of $r \times t$ for $\eta=10^{-2}$ and for two different values of $V_{0}$, namely: (a) $V_{0}$ $=10^{-3}$ and (b) $V_{0}=10^{-2}$. The control parameter $\delta$ is labeled in the figure. Since in the process of motion the particle may acquires small values of the velocity, then depending on the coordinate angle of the particle's trajectory, all of its energy may dissipate, thus stopping the dynamics. Such a behavior is not observed for values of $\delta>1.48$.

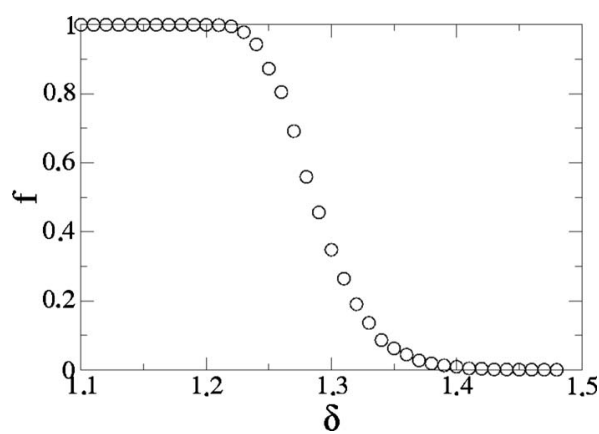

FIG. 8. Plot of $f$ versus $\delta$. For $\delta>1.48$, none of initial conditions have their initial energy completely dissipated up to $10^{5} \mathrm{col}-$ lisions with the boundary. The control parameters used were $p=3$, $\epsilon=0.1$, and $a=0.1$ and the damping coefficient $\eta=10^{-3}$.

We also evaluated the fraction of initial conditions, $f$, which reaches the rest as function of the control parameter $\delta$. This fraction is obtained by evolving a grid of $100 \times 100$ different initial conditions $\alpha_{0} \in[0, \pi]$ and $\theta_{0} \in[0,2 \pi]$ for an initial velocity $V_{0}=1$ and random $t_{0} \in[0,2 \pi]$ for a fixed damping coefficient $\eta=10^{-3}$ and the fixed control parameters $p=3, \epsilon=0.1$ and $a=0.1$. When the particle stops, then a new initial condition is taken. Since the simulations are very computational time consuming, we impose some criteria to stop evolving the orbit. If the particle did not stop after $10^{5} \mathrm{col}-$ lisions with the boundary, a new initial condition is started and the previous orbit is not used to compute $f$. A plot of $f$ versus $\delta$ is shown in Fig. 8. If $\delta<1.2$, then the particles always stop. Moreover, for $1.2<\delta<1.48$, there is a monotonic decay of $f$ and for $\delta>1.48$, no initial conditions have their total energy dissipated up to $10^{5}$ collisions with the boundary.

The limits of the relations Eqs. (17) and (18) for both $\delta$ $\rightarrow 1$ and $\delta \rightarrow 2$ can be obtained. Expanding Eq. (17) for $\delta$ $\rightarrow 1$ and taking only the first order, we have that

$$
V(t)=V_{n} \exp \left[-\eta\left(t-t_{n}\right)\right]+\bigcirc(\delta-1),
$$

while the same procedure for Eq. (18) gives that

$$
r(t)=\frac{V_{n}}{\eta}\left[1-e^{-\eta\left(t-t_{n}\right)}\right]+\bigcirc(\delta-1) .
$$

We see that both Eqs. (19) and (20) recover the case of linear damping force and therefore lead to linear decay of the velocity, as demonstrated before.

On the other hand, for $\delta \rightarrow 2$, the expansions of Eqs. (17) and (18) give that

$$
\begin{gathered}
V(t)=\frac{V_{n}}{1+\eta\left(t-t_{n}\right)}+\bigcirc(\delta-2), \\
r(t)=\frac{1}{\eta} \ln \left[1+\eta V_{n}\left(t-t_{n}\right)\right]+\bigcirc(\delta-2),
\end{gathered}
$$

which lead therefore to an exponential decay in the velocity of the particle for $\delta \rightarrow 2$, as shown before. For different values of $\delta$, say $1<\delta<2$, the decay of the velocity is generally 


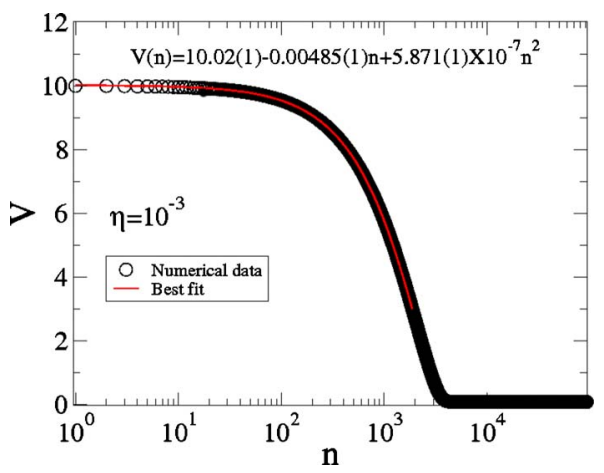

FIG. 9. (Color online) Decay of the velocity of the particle for $\delta=1.5$. The control parameters used were $p=3, \epsilon=0.1$, and $a=0.1$ and the damping coefficient $\eta=10^{-3}$. The best fitting, by the second degree polynomial is $V(n)=10.02(1)-0.00485(1) n+5.871(1)$ $\times 10^{-7} n^{2}$.

a power-like, as one can see in Fig. 9 for $\delta=1.5$. The damping coefficient used was $\eta=10^{-3}$ and the best fitting, given by a second order polynomial in $n$ is $V(n)=10.02(1)$ $-0.00485(1) n+5.871(1) \times 10^{-7} n^{2}$.

Let us now extend the procedure used in Sec. III B to describe the behavior of the average velocity and hence the critical exponents that describe the transition from limited to unlimited energy growth. According to Fig. 8, termination of the dynamics is observed for $\delta<1.48$. Since we want characterize the phase transition from limited to unlimited energy growth, the range considered is $\delta \geq 1.5$ and termination of the dynamics is avoided. A plot of the average velocity against $n$ for $\delta=1.99$ is shown in Fig. 10 . We see that average velocity behaves quite similar to the case described in Sec. III B and that the scaling hypotheses also are the same as those described by Eqs. (14)-(16). However, the critical exponents obtained are rather different and depend on $\delta$. We have considered seven different values of $\delta$ and obtained the critical exponents $\gamma$ and $z$, as shown in Table I. Extensive numerical simulations were carried out moreover leading to an average $\bar{\beta}=0.480(3) \cong 0.5$. To confirm the validity of the scaling hypotheses, universal plots were obtained, as shown in Fig. 11 for different values of $\delta$ and at least three different values of $\eta$. A plot of the critical exponents as function of the control parameter $\delta$ is shown in Fig. 12. We see that as the control parameter $\delta$ rises, both critical

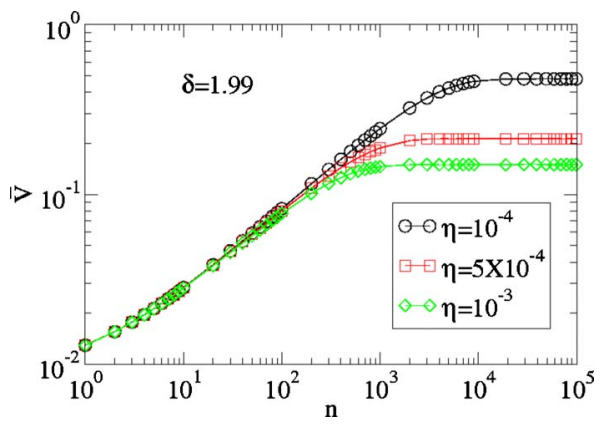

FIG. 10. (Color online) Plot of $\bar{V}$ versus $n$ for $\delta=1.99$ and different values of $\eta$, as labeled in the figure.
TABLE I. Critical exponents $\gamma$ and $z$ obtained for different values of $\delta$, as labeled in the Table.

\begin{tabular}{lcc}
\hline \hline$\delta$ & $\gamma$ & $z$ \\
\hline 1.5 & $-0.659(2)$ & $-1.368(6)$ \\
1.6 & $-0.616(2)$ & $-1.275(7)$ \\
1.7 & $-0.582(1)$ & $-1.211(5)$ \\
1.8 & $-0.5511(9)$ & $-1.151(3)$ \\
1.9 & $-0.5246(6)$ & $-1.103(3)$ \\
1.95 & $-0.5115(6)$ & $-1.061(2)$ \\
1.99 & $-0.5017(5)$ & $-1.047(2)$ \\
2.0 & $-0.5005(4)$ & $-1.027(1)$ \\
\hline \hline
\end{tabular}

exponents exhibit a monotonically growth. As $\delta \rightarrow 2$, the critical exponent $\gamma \rightarrow-0.5$ and $z \rightarrow-1$. The exponents shown in Table I, thus, confirm that FA will take place in the limit of vanishingly dissipation and that such a transition is smooth.

\section{CONCLUDING REMARKS}

We have studied some dynamical properties of billiards in a driven oval-like shaped domain under three different types of a drag force. For a damping force proportional to the particle's velocity, we have shown that the particle experiences a linear decay on the velocity and that the speed of the
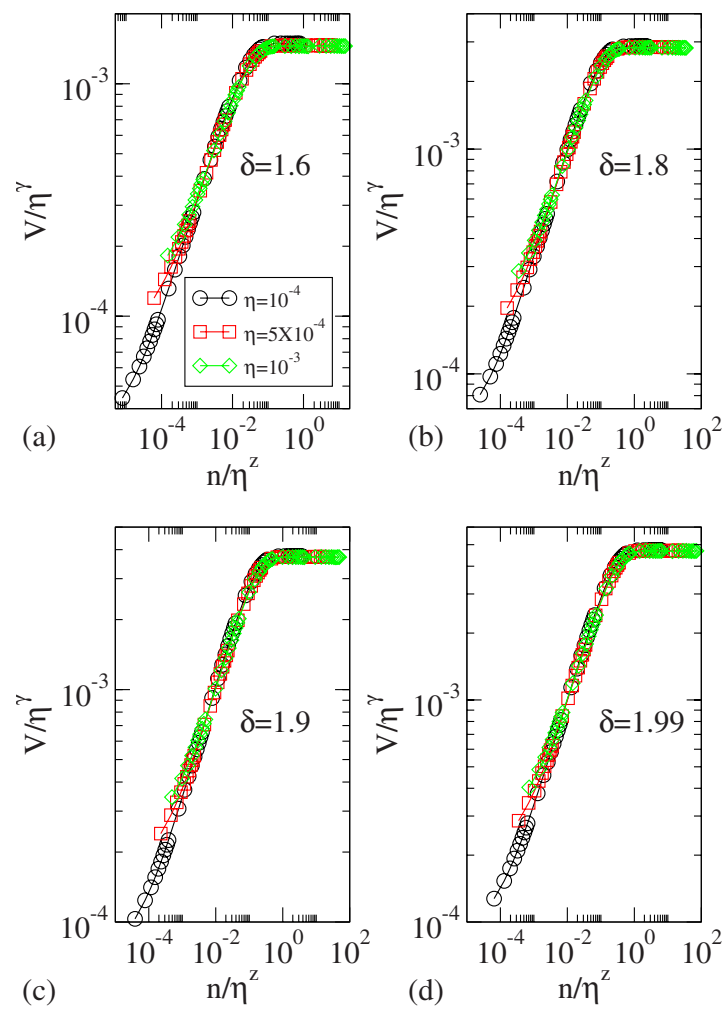

FIG. 11. (Color online) Universal plot of $\bar{V}$ versus $n$, after a suitable rescale on both the axis. The control parameters used were: (a) $\delta=1.6$; (b) $\delta=1.8$; (c) $\delta=1.9$; and (d) $\delta=1.99$. The critical exponents used on the rescale were those shown in Table I. 

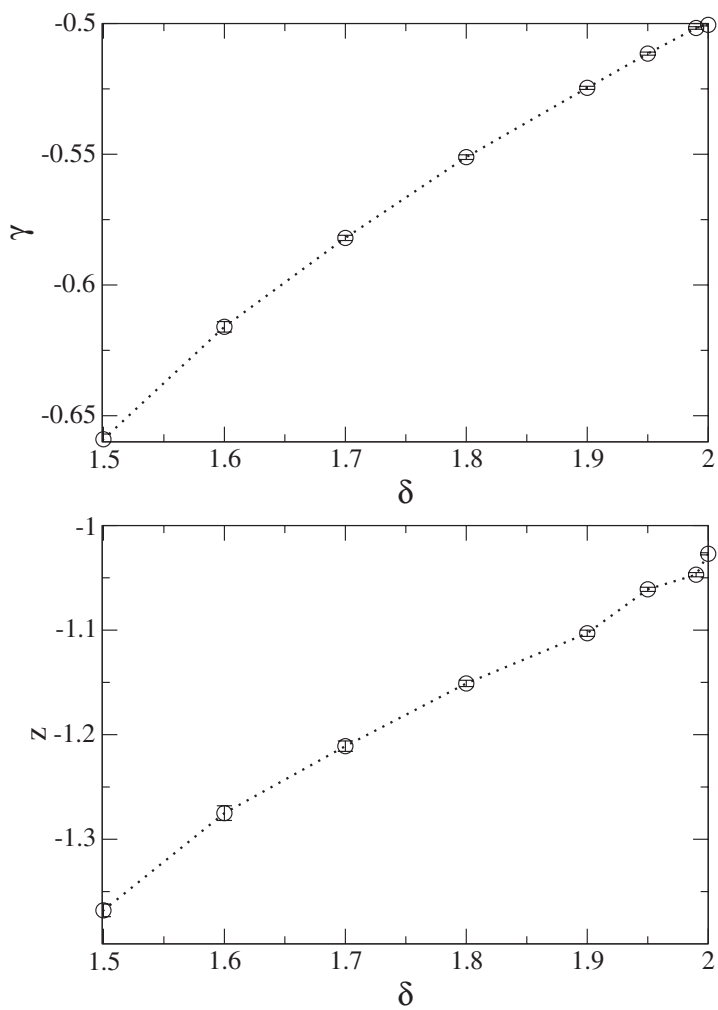

FIG. 12. Plot of the critical exponents as function of $\delta$. (a) $\gamma$ versus $\delta$; (b) $z$ versus $\delta$.

decay is proportional to $-1.5 \eta$, thus far slow as compared to the Fermi-Ulam model [32]. Our results therefore confirm that FA is suppressed and the particle stops. For the case of a drag force proportional to a square of the velocity of the particle, our results confirm that FA also is suppressed. The decay of the particle's velocity is of an exponential type with the exponent of the order of $-1.5 \eta$, therefore also slower than in the Fermi-Ulam model [33]. As the velocity of the particle decreases, the damping force decays faster and the particle never stops. We have shown that, starting with a small energy, the velocity of the particle could be described with the help of scaling. As the damping coefficient $\eta \rightarrow 0$, the particle experiences a phase transition from limited to unlimited energy growth. The critical exponents obtained are the same as those for the dissipative bouncer model $[37,38]$. Despite the differences of the two models, the criticality near such a phase transition is the same. Therefore, near this phase transition, the two models belong to the same class of universality. As the control parameter $\delta$ varies, the particle can stops. The fraction of initial conditions for which it happens was computed as function of $\delta$ for an ensemble of $10^{4}$ initial conditions. For $\delta<1.2$, all the initial conditions lead the dynamics to stop. However, for $1.2<\delta<1.48$, there is a monotonic decay of $f$ while for $\delta$ $>1.48$, none of the initial conditions have their total energy dissipated in the process of numerical simulations, i.e., up to $10^{5}$ collisions with the boundary. Critical exponents characterizing a phase transition from limited to unlimited energy growth were also obtained from different values of $\delta$, as shown in Table I.

\section{ACKNOWLEDGMENTS}

The work of E.D.L. was partially supported by CNPq, FAPESP, and FUNDUNESP, Brazilian agencies. He also acknowledges support for a visit to Georgia Tech from comissão mista CAPES/FULBRIGHT. L.B. was partially supported by NSF (Grant No. DMS-0900945).
[1] N. Chernov and R. Markarian, Chaotic Billiards (American Mathematical Society, Providence, 2006), Vol. 127.

[2] M. V. Berry, Eur. J. Phys. 2, 91 (1981).

[3] L. A. Bunimovich, Commun. Math. Phys. 65, 295 (1979).

[4] D. Sweet et al., Physica D 154, 207 (2001).

[5] H. D. Graf, H. L. Harney, H. Lengeler, C. H. Lewenkopf, C. Rangacharyulu, A. Richter, P. Schardt, and H. A. Weidenmuller, Phys. Rev. Lett. 69, 1296 (1992).

[6] T. Sakamoto et al., Jpn. J. Appl. Phys. 30, L1186 (1991).

[7] J. P. Bird, J. Phys.: Condens. Matter 11, R413 (1999).

[8] E. Persson, I. Rotter, H. J. Stockmann, and M. Barth, Phys. Rev. Lett. 85, 2478 (2000).

[9] E. D. Leonel, Phys. Rev. Lett. 98, 114102 (2007).

[10] J. Stein and H. J. Stockmann, Phys. Rev. Lett. 68, 2867 (1992).

[11] H. J. Stökmann, Quantum Chaos: An Introduction (Cambridge University Press, Cambridge, England, 1999).

[12] V. Milner, J. L. Hanssen, W. C. Campbell, and M. G. Raizen, Phys. Rev. Lett. 86, 1514 (2001).

[13] N. Friedman, A. Kaplan, D. Carasso, and N. Davidson, Phys. Rev. Lett. 86, 1518 (2001).

[14] M. F. Andersen, T. Grunzweig, A. Kaplan, and N. Davidson,
Phys. Rev. A 69, 063413 (2004).

[15] M. F. Andersen, A. Kaplan, T. Grunzweig, and N. Davidson, Phys. Rev. Lett. 97, 104102 (2006).

[16] C. M. Marcus, A. J. Rimberg, R. M. Westervelt, P. F. Hopkins, and A. C. Gossard, Phys. Rev. Lett. 69, 506 (1992).

[17] E. Fermi, Phys. Rev. 75, 1169 (1949).

[18] A. Y. Loskutov, A. B. Ryabov, and L. G. Akinshin, J. Exp. Theor. Phys. 89, 966 (1999).

[19] P. J. Holmes, J. Sound Vib. 84, 173 (1982).

[20] A. K. Karlis, P. K. Papachristou, F. K. Diakonos, V. Constantoudis, and P. Schmelcher, Phys. Rev. Lett. 97, 194102 (2006); Phys. Rev. E 76, 016214 (2007).

[21] A. J. Lichtenberg and M. A. Lieberman, Regular and Chaotic Dynamics, Applied Mathematical Sciences (Springer-Verlag, New York, 1992), Vol. 38.

[22] D. G. Ladeira and Jafferson Kamphorst Leal da Silva, Phys. Rev. E 73, 026201 (2006).

[23] A. Loskutov and E. D. Leonel, Math. Probl. Eng. (2009), 848619.

[24] A. Loskutov, A. B. Ryabov, and L. G. Akinshin, J. Phys. A 33, 7973 (2000).

[25] F. Lenz, F. K. Diakonos, and P. Schmelcher, Phys. Rev. Lett. 
100, 014103 (2008).

[26] D. F. M. Oliveira and E. D. Leonel, Physica A 389, 1009 (2010).

[27] E. D. Leonel and L. A. Bunimovich, Phys. Rev. Lett. 104, 224101 (2010).

[28] A. Arroyo, R. Markarian, and D. P. Sanders, Nonlinearity 22, 1499 (2009).

[29] D. F. M. Oliveira and E. D. Leonel, Commun. Nonlinear Sci. Numer. Simul. 15, 1092 (2010).

[30] S. O. Kamphorst, E. D. Leonel, and J. K. L. da Silva, J. Phys. A: Math. Theor. 40, F887 (2007).

[31] E. D. Leonel, D. F. M. Oliveira, and A. Loskutov, Chaos 19, 033142 (2009).

[32] E. D. Leonel and P. V. E. McClintock, Phys. Rev. E 73,
066223 (2006); J. Phys. A 39, 11399 (2006).

[33] E. D. Leonel and D. F. Tavares, CP913, Nonequilibrium Statistical Mechanics and Nonlinear Physics, XV Conference, edited by O. Descalzi, O. A. Rosso, and H. A. Larrondo, (American Institute of Physics, New York, 2007), Vol. 108.

[34] M. Robnik and M. V. Berry, J. Phys. A 18, 1361 (1985).

[35] M. V. Berry and E. C. Sinclair, J. Phys. A 30, 2853 (1997).

[36] M. Aichinger, S. Janecek, and E. Rasanen, Phys. Rev. E 81, 016703 (2010).

[37] E. D. Leonel and A. L. P. Livorati, Physica A 387, 1155 (2008).

[38] Andre Luis Prando Livorati, D. G. Ladeira, and E. D. Leonel, Phys. Rev. E 78, 056205 (2008). 